\title{
The role of the quantum dispersion in the Coulomb correction of Bose-Einstein correlations
}

\author{
H. Merlitz ${ }^{a, b}$ and D. Pelte ${ }^{a, c}$ \\ a: Physikalisches Institut der Universität Heidelberg, D-69120 Heidelberg \\ b: Gesellschaft für Schwerionenforschung (GSI) Darmstadt, Germany \\ c: Max-Planck-Institut für Kernphysik, D-69117 Heidelberg
}

\begin{abstract}
The time dependent Schrödinger equation for two identical and charged pions is solved using wavepacket states. It is shown that the expected Coulomb distortion in the momentum correlation function is obliterated by the dispersion of the localized states, and therefore becomes unobservable.
\end{abstract}

PACS: 25.70.-z

Keywords: HBT-Analysis; Coulomb correction; Wavepackets

E-mail: pelte@pel5.mpi-hd.mpg.de 


\section{Introduction}

The Bose-Einstein correlations of identical charged pions are believed to be distorted by the Coulomb interaction between both pions. Since the end of the seventies, several efforts where made to investigate these distortions. A reliable information about the size of the pion source can only be extracted from the correlation signal when the properties of the Coulomb distortions are sufficiently understood. The size of the Coulomb effect, and how it should be calculated, was always a source of intensive discussions. In the early theoretical approaches, the square of the relative Coulomb wave function for a pointlike source, the Gamow factor, was employed to obtain the Coulomb correction of the correlation function [回]. Later it was realized that this ansatz overestimated the Coulomb suppression, and since then different models where developed which successively led to smaller and smaller distortions. First, the finite source size was included [2], later the influence of the exchange terms [3], the influence of long-lived resonances [4], of Coulomb screening due to other charged particles [5] and the finite momentum resolution of the detector [6].

Already in 1986, however, it became clear that a correct treatment of pions in the fireball must include their localization in terms of quantum mechanical wavepackets, the spatial widths of which are connected to the mean free path of the pions which has a value of a few fm [7]. Recently, a consistent wavepacket description was presented by the authors and applied to different numerical codes [8]. A somewhat different ansatz, which may be suitable especially for very high multiplicities, is developed in [9]. It was demonstrated, how the localization of the pions introduces a zero point energy, which consequently leads to a momentum uncertainty in the pion ensemble, i. e. to dispersion. In order to estimate the possible influence of the dispersion onto the Coulomb effects, we compare the size of the Coulomb potential with the zero point energy using a simple picture: Imagine two pions which are separated by the distance $r_{\mathrm{o}}$. Both states shall not overlap significantly, so that we require them to be localized in Gaussian wavepackets with the spatial extension $\sigma_{\mathrm{o}}=r_{\mathrm{o}} / 2$. We evaluate the Coulomb energy $E_{\text {coul }}=e^{2} / r_{\mathrm{o}}$ and the zero point (quantum-) energy $E_{\mathrm{q}}=3 \hbar^{2} /\left(8 m \sigma_{\mathrm{o}}^{2}\right)$ for a distance of $r_{\mathrm{o}}=4 \mathrm{fm}$ and obtain $E_{\text {coul }} \approx 0.4 \mathrm{MeV}$ and $E_{\mathrm{q}} \approx 26 \mathrm{MeV}$. Therefore, the quantum energy $E_{\mathrm{q}}$ is nearly two orders of magnitude larger than $E_{\text {coul }}$. Only for distances $r_{\mathrm{o}}>290 \mathrm{fm}$ exceeds the Coulomb energy the localization energy and the picture of two classical particles with Coulomb repulsion becomes valid. For smaller distances the dispersion introduces a strong random motion into the ensemble which obliterates the directed motion caused by the Coulomb repulsion. This suggests that a correct quantum mechanical treatment of the Coulomb interaction is necessary and can not be substituted by any semiclassical model. 


\section{Matrix representation of the Schrödinger equa- tion}

The Schrödinger equation for two identical charged bosons,

$$
\hat{H}\left|\Psi\left(\mathbf{r}_{1}, \mathbf{r}_{2}, t\right)\right\rangle=i \hbar \partial_{t}\left|\Psi\left(\mathbf{r}_{1}, \mathbf{r}_{2}, t\right)\right\rangle,
$$

with the Hamiltonian

$$
\hat{H}=-\frac{\hbar^{2}}{2 m}\left(\Delta_{1}+\Delta_{2}\right)+\frac{e^{2}}{\left|\mathbf{r}_{1}-\mathbf{r}_{2}\right|^{2}} \equiv \hat{T}+\hat{V}
$$

and the symmetrized 2- particle state

$$
\left|\Psi\left(\mathbf{r}_{1}, \mathbf{r}_{2}, t\right)\right\rangle=\mathcal{N}_{2}\left(\left|\chi_{1}\left(\mathbf{r}_{1}, t\right)\right\rangle\left|\chi_{2}\left(\mathbf{r}_{2}, t\right)\right\rangle+\left|\chi_{2}\left(\mathbf{r}_{1}, t\right)\right\rangle\left|\chi_{1}\left(\mathbf{r}_{2}, t\right)\right\rangle\right)
$$

has to be solved. Since the Hamiltonian does not depend on time, the solution could be found on principle by time independent methods. The problem, however, are the initial conditions of the system: At $t=0$ each particle is localized within a certain volume (wavepackets in the fireball). There exists neither an analytical solution for this problem nor a way how to transform these initial conditions into appropriate border conditions for a time independent treatment. We therefore have to solve the time dependent problem. To start with, we expand the state (3) into a set of basis functions

$$
\left|\Psi\left(\mathbf{r}_{1}, \mathbf{r}_{2}, t\right)\right\rangle=\sum c_{k}(t)\left|\Psi_{k}\left(\mathbf{r}_{1}, \mathbf{r}_{2}, t\right)\right\rangle
$$

with the c-valued coefficients

$$
c_{k}(t)=\left\langle\Psi_{k}\left(\mathbf{r}_{1}, \mathbf{r}_{2}, t\right) \mid \Psi\left(\mathbf{r}_{1}, \mathbf{r}_{2}, t\right)\right\rangle .
$$

Now the Schrödinger equation can be written in matrix form

$$
i \hbar \dot{\mathbf{c}}=\tilde{S}^{-1}(\tilde{H}-\tilde{D}) \mathbf{c}
$$

with the time dependent coefficient vector c , the Hamilton matrix $\tilde{H}_{i j}=\left\langle\Psi_{i}|\hat{T}+\hat{V}| \Psi_{j}\right\rangle$, the overlap matrix $\tilde{S}_{i j}=\left\langle\Psi_{i} \mid \Psi_{j}\right\rangle$ and the time evolution matrix $\tilde{D}_{i j}=\left\langle\Psi_{i}\left|i \hbar \partial_{t}\right| \Psi_{j}\right\rangle$. This representation of the Schrödinger equation is equivalent to Eq. (11) if the basis provides a complete set of states in the 2- particle Hilbert space. In any numerical calculations, of course, only a limited number of basis functions can be used so that an adequate choice of the basis is essential in order to reach the required accuracy and, at the same time, to keep the numerics within affordable limits.

\section{Choice of the basis set}

For the single particle states we use the following time dependent wave functions:

$$
\chi_{\vec{l}_{j} k}(\mathbf{r}, t)=x_{k}^{l_{j x}} y_{k}^{l_{j y}} z_{k}^{l_{j z}} \exp \left(\frac{i}{\hbar}\left(\mathbf{P}_{k}(t) \cdot \mathbf{r}-\theta_{\vec{l}_{j}, k}(t)\right)-\frac{\left(\mathbf{r}-\mathbf{R}_{k}(t)\right)^{2}}{4 s(t) \sigma_{\mathrm{o}}}\right)
$$


with the phase

$$
\theta_{\vec{l}_{j}, k}(t)=\int_{0}^{t} E_{k}^{\mathrm{kin}}(\tau) d \tau+\hbar\left(l_{j x}+l_{j y}+l_{j z}+3 / 2\right) \arctan \left(\frac{\hbar t}{2 m \sigma_{o}^{2}}\right)
$$

and $x_{k}^{l_{j x}}=\left(\mathbf{r}_{x}-\mathbf{R}_{k_{x}}\right)^{l_{j x}}$, and similar for the $y$ - and $z$ coordinates. The capital coordinates $\left(\mathbf{R}_{k}(t), \mathbf{P}_{k}(t)\right)$ denote the phase space coordinates of the $k$ 'th wave packet centre, whereas $\mathbf{r}$ is the free variable of the state in configuration space representation. The time dependent width is given by $s(t)=\sigma_{\mathrm{o}}+i \hbar t /\left(2 m \sigma_{\mathrm{o}}\right)$ and the initial width is $\sigma_{\mathrm{o}}$. With the index vector $\vec{l}_{j}=(0,0,0)$ we get the usual Gaussian wave packet which was already used in [8]. Following the notation used in the harmonic oscillator problem, this function may be called "s- type" function, and consequently the index vector $\overrightarrow{l_{j}}=(1,0,0)$ defines a "p- type" function and so on. We now build symmetrized 2particle states from the single particle states, because then the basis functions already fulfill the correct permutation symmetry. An additional symmetry requirement is that the squared amplitude must be symmetric with respect to the centre of mass of the system, which requires the index vectors of both single particle states to be equal. We therefore define the basis in terms of the homogeneous states

$$
\Psi_{\overrightarrow{l_{i}}} \sim \chi_{\vec{l}_{i} 1}\left(\mathbf{r}_{1}, t\right) \chi_{\vec{l}_{i} 2}\left(\mathbf{r}_{2}, t\right)+\chi_{\vec{l}_{i} 2}\left(\mathbf{r}_{1}, t\right) \chi_{\vec{l}_{i} 1}\left(\mathbf{r}_{2}, t\right)
$$

We use a set of 2- particle basis functions, which contains $1 \mathrm{~s}$ - function, 3 p- functions, $6 \mathrm{~d}$ - functions and $10 \mathrm{f}$-functions. The use of even larger basis sets is prohibited by the exponentially increasing numerical effort. For example, the evaluation of a complete trajectory (which typically requires 500 timesteps) needs 1 minute for a sp- basis, $1 / 2$ hour for a spd- basis and 1/2 day for a spdf- basis

\section{The solution of Schrödinger's equation}

In order to solve the Schrödinger equation (6), the matrices $\tilde{V}, \tilde{T}, \tilde{D}$ and $\tilde{S}$ have to be evaluated. It is possible to obtain analytical expressions for the kinetic energy- and time evolution matrix elements, whereas the potential matrix elements, representing a 6- fold integral, can be reduced to an one dimensional integration and tabulated. Details of the very lengthy calculations may not be given here. We refer to the literature in quantum chemistry?t.

At the beginning of each event, the system has to be initialized. As source function we used the following simple parametrization of the phase space:

$$
\rho(\mathbf{R})=\frac{1}{\left(\pi R_{s}^{2}\right)^{\frac{3}{2}}} \exp \left(\frac{-\mathbf{R}^{2}}{R_{s}^{2}}\right) \quad \text { and } \quad f(\mathbf{P})=(2 \pi m T)^{-3 / 2} \exp \left(\frac{-\mathbf{P}^{2}}{2 m T}\right)
$$

\footnotetext{
${ }^{1}$ Values obtained on a DEC $250^{4 / 266}$ workstation.

${ }^{2}$ For example, in [10], Chapter 6 , the calculations are done for the time independent Gaussians. For the evaluation of our time dependent basis set, a detailed script (13 pages) can be obtained from the authors.
} 
i. e. the source is a static Gaussian with rms- radius $\sqrt{3 / 2} R_{s}$ and temperature $T$. This choice is motivated by the fact that an analytical expression for the 2- particle correlation function is available for noninteracting wavepackets [8]:

$$
\mathcal{C}_{2}\left(\mathbf{p}_{1}, \mathbf{p}_{2}\right)=\sum_{k=0}^{\infty}(-1)^{k}\left(\frac{a_{o}^{2} b_{o}^{2}}{a_{k} b_{k} c_{k} d_{k}}\right)^{3 / 2}\left(e^{f_{k}\left(\mathbf{p}_{1}, \mathbf{p}_{2}\right)}+e^{g_{k}\left(\mathbf{p}_{1}, \mathbf{p}_{2}\right)}\right)
$$

with

$$
\begin{aligned}
& f_{k}\left(\mathbf{p}_{1}, \mathbf{p}_{2}\right)=-\left(\mathbf{p}_{1}-\mathbf{p}_{2}\right)^{2} \frac{k}{16 \sigma_{p}^{6} a_{k} c_{k}} \\
& g_{k}\left(\mathbf{p}_{1}, \mathbf{p}_{2}\right)=-\left(\mathbf{p}_{1}-\mathbf{p}_{2}\right)^{2}\left(\frac{4 \sigma_{p}^{2} a_{k}+k}{32 \sigma_{p}^{6} a_{k} c_{k}}+\frac{1}{2 \hbar^{2} R_{s}^{2} b_{k} d_{k}}\right)
\end{aligned}
$$

and

$$
\begin{aligned}
& a_{k}=\frac{1}{2 \sigma_{p}^{2}}+\frac{1}{2 m T}+\frac{k}{4 \sigma_{p}^{2}}, \quad b_{k}=\frac{1}{R_{s}^{2}}+\frac{k}{4 \sigma_{o}^{2}} \\
& c_{k}=a_{k}-\frac{k^{2}}{16 \sigma_{p}^{4} a_{k}}, \quad d_{k}=b_{k}-\frac{k^{2}}{16 \sigma_{o}^{4} b_{k}}
\end{aligned}
$$

$\sigma_{\mathrm{p}}=\hbar /\left(2 \sigma_{\mathrm{o}}\right)$ is the width of the wavepacket in momentum space. The state was initialized to be a pure s- state, i. e. with the coefficient vector $\mathbf{c}(t=0)=(1,0, \ldots, 0)$ in Eq. (6), and the initial width of the Gaussian was $\sigma_{\mathrm{o}}=1.8 \mathrm{fm}$. The time propagation was performed using a second order differential scheme [11]. For each timestep, the force between both wavepackets was obtained numerically using $\dot{\mathbf{P}}_{2} \equiv-\nabla_{21}\left\langle\Psi\left|\hat{V}\left(\mathbf{R}_{21}\right)\right| \Psi\right\rangle$ and $\dot{\mathbf{P}}_{1}=-\dot{\mathbf{P}}_{2}$. Here, $\mathbf{R}_{21}$ is the difference vector between both centres. The propagation was stopped when the residual Coulomb energy had dropped below $5 \%$ of the initial value. At the end of each event, the final state was Fourier transformed into the momentum space representation. Such a state defines a quantum mechanical (pure) ensemble of equally prepared systems. We can extract an arbitrary number of representatives by using the squared amplitude as density function and applying the Monte Carlo procedure presented in $[8]$ to sample out a number of $N_{s}$ correlated pairs. The 2particle correlation function, however, is built by the mixed ensemble which is obtained by averaging over the source function. Therefore we have to calculate a large number $N_{e}$ of events with different initial conditions according to the source densities Eq. (10), yielding $N_{e} \cdot N_{s}$ pairs which finally obey the correct mixed statistics.

\section{Results}

The source parameters were set to $R_{s}=5 \mathrm{fm}$ and $T=50 \mathrm{MeV}$. In order to gain an intuitive feeling about the strength of the Coulomb effect, "pions" with different charges $Z=1, Z=3$ and $Z=5$ were simulated. Since the Coulomb potential 
between both particles increases quadratically with $Z$, this set covers a range between the realistic case and the case where the Coulomb energy nearly reaches the same magnitude as the localization energy as was discussed in Sect. 1. $N_{e}=2000$ events were generated using a sp- basis set, the average system propagation time was $250 \mathrm{fm} / \mathrm{c}$ until the Coulomb potential had decayed below $5 \%$ of its initial value. Table 1 displays some key parameters for the different systems. Clearly the average initial Coulomb energy increases quadratically with the particle charge (second column). The third column demonstrates that around $70 \%$ of this initial Coulomb energy is converted into kinetic energy of the wavepackets, independent of the charge. This implies that the residual $30 \%$ are put into excitation of the p- basis functions, i. e. into deformation of the wavepackets. Since with the sp- basis we only have 4 basis functions which are orthogonal, we can use the fact that

$$
\left|c_{s}\right|+\left|c_{p_{x}}\right|+\left|c_{p_{y}}\right|+\left|c_{p_{z}}\right|=1
$$

to compare the weights that are present in the final state. The fourth column shows that in case of $Z=1$ in the average only $0.4 \%$ of the weight is deposited into the pbasis, whereas $99.6 \%$ remain in the s- function. This implies that the state remains nearly undisturbed. In case of $Z=5$ the situation is different: Already $8 \%$ of the weight is put into the p- functions, in $10 \%$ of the events this weight even exceeds $20 \%$. A considerable deformation is therefore induced by the Coulomb force.

For the evaluation of the 2- particle correlation function, each of the 2000 generated events was used to extract $N_{s}=5000$ correlated pairs, yielding a statistics of $10^{7}$ pairs. Figure 11 displays the resulting correlation functions. Two facts are of importance:

1. For $Z=1$ the distortion of the correlation function is invisible.

2. For $Z=3$ and $Z=5$ an increasing deviation from the undistorted correlation appears, but the effect is global in the sense that the whole correlation function is involved rather than only the part of low momentum differences.

Several test simulations were performed with different basis sets including sp-, spdand spdf- sets in order to check whether the used sp- basis is already sufficiently large. For the $Z=1$ simulation no differences were observed, which implies that the spbasis is sufficient to yield reliable results. This is not surprising since the deformation of the wavepackets is very small as discussed above. For the $Z=5$ simulation some dependence on the basis size could be observed. The size of these effects suggests that a simulation with spdf- basis instead the sp- basis may lead to further changes of the correlation function, which may be as large as $20 \%$ of the distortions calculated with the smaller basis. Such simulations, however, could not be performed with sufficient statistics due to the huge numerical effort.

As a second time dependent method, the molecular dynamics procedure which was discussed in [8] and is based on Bohm's quantum theory of motion, was employed to evaluate the pion-pion Coulomb interaction. In this method, only the Gaussian s- functions are used. The additional degree of freedom is included by means of an 
equation of motion for certain test particles, which contains the quantum potential and accounts for dispersion- and correlation effects. Since it is the distribution of the test particles which is used to deduce the correlation function, the deformation of the wavepacket is achieved indirectly due to their Coulomb- distorted positions. This ansatz is approximate in the sense that the distorted test particles lead to distorted density distributions and therefore to a distorted state, a fact that in an exact treatment would change the quantum forces and therefore the particle trajectories and again (in higher order) the state. But for only small distortions it was found to be sufficient to evaluate the quantum force using the undisturbed state.

The simulations were repeated for the $Z=1$ and $Z=5$ case. A number of $3 \cdot 10^{6}$ pion pairs were generated for each charge and used to determine the correlation functions. For $Z=1$ we obtained the same result as with the sp- basis expansion, namely no visible Coulomb distortion. Figure 2 only displays the result of the $Z=5$ simulation in comparison to the corresponding result from the basis expansion. An overall qualitative agreement is observed, for small momentum differences, however, there exist some deviations. This indicates the possible inaccuracy of both methods, since for the hypothetical and rather extreme case $Z=5$ the Coulomb effects are no longer small and both methods begin to suffer from the approximative character of the small basis respective the unproper evaluation of the quantum force. For the $Z=1$ simulation, on the other hand, the approximations are well justified and the calculations suggest that the pion-pion Coulomb interaction has no visible effect onto the 2- particle correlation function and therefore experimental data must not be "corrected" to remove a nonexistent effect.

\section{$6 \quad$ Why scattering theory fails}

Usually, the pion-pion Coulomb effects are treated by considering the time independent scattering of plane waves in a Coulomb potential. The shortcomings of this ansatz are most obvious if we write the correct quantum mechanical ansatz in the Bohm formalism. We may initialize a wavepacket of width $\sigma_{\mathrm{o}}$ with the centre coordinate $\mathbf{R}_{\mathrm{o}}$ and a test particle at the position $\mathbf{x}_{\mathrm{o}}$ and obtain the following equation of motion for the test particle:

$$
m \frac{d^{2} \mathbf{x}}{d t^{2}}=-\nabla(V+Q)
$$

with the quantum potential

$$
Q(t=0)=\frac{\hbar^{2}}{4 m \sigma_{\mathrm{o}}^{2}}\left(3-\frac{\left(\mathbf{x}_{\mathrm{o}}-\mathbf{R}_{\mathrm{o}}\right)^{2}}{2 \sigma_{\mathrm{o}}^{2}}\right),
$$

which exerts a repulsive force onto the test particle and which when integrated over the density distribution yields the zero point energy of the wavepacket [8]. In the plane wave limit $\left(\sigma_{\mathrm{o}} \rightarrow \infty\right)$, the quantum potential vanishes and (16) becomes a classical equation of motion. This is the reason why the results from scattering theory can be 
made to agree with pure classical trajectory calculations [5]. As a crosscheck, we did repeat the $Z=1$ simulation for wavepackets without dispersion, i. e. for a fixed width. The resulting correlation function is in agreement with the predictions of the classical "toy model" [5], which proves that it is the dispersion which masks the Coulomb effect.

In conclusion, we have shown that the correlation functions in pion interferometry are not distorted by pion-pion Coulomb effects. Due to the initial conditions, which force the pions to be localized within a small volume of a few $\mathrm{fm}^{3}$, the zero point energy produces a dispersion which makes the comparably tiny distortion due to Coulomb interaction to become unobservable. For heavier particles the strength of the dispersion decreases and therefore then the Coulomb effect is expected to be of more relevance. We have supported our argumentation by numerical simulations using two different methods. In the first (basis expansion), the Coulomb influence is accounted for on the level of the state amplitude, in the other (Bohm's molecular dynamics) on the level of the equation of motion. Scattering theory fails to produce the correct results, because

with the removal of the quantum potential in Eq. (16) the most important part of the dynamical evolution is neglected. This implies that experimental data, which are published after "Coulomb correction", are wrong for small momentum differences.

\section{References}

[1] M. Gyulassy, S. K. Kauffmann and L. W. Wilson, Phys. Rev. C 20 (1979) 2267.

[2] M. G. Bowler, Phys. Lett. B 270 (1991) 69.

[3] M. Biyajima, T. Mizoguchi, T. Osada and G. Wilk, Phys. Lett. B 353 (1995) 340.

[4] Yu. M. Sinyukov, S. V. Akkelin and A. Yu. Tolstykh, Nucl. Phys. A 610 (1996) 278c.

[5] G. Baym and P. Braun-Munzinger, Nucl. Phys. A 610 (1996) 286c.

[6] J. Barrette and the E877 Collaboration, Phys. Rev. Lett. 78 (1997) 2916.

[7] S. Pratt, Phys. Rev. D 33 (1986) 72.

[8] H. Merlitz and D. Pelte, Z. Phys. A 357 (1997) 175.

[9] J. Zimanyi and T. Csörgö, hep-ph/9705432 v2 (1997), submitted to Phys. Rev. C.

[10] E. Clementi, "Modern Techniques in Computational Chemistry: MOTECC89", ESCOM Science Publishers B. V. (1989).

[11] C. Leforestier et al., Journal of Computational Physics 94, 59 (1991). 
Table 1: Averaged values for the initial Coulomb energy (2'nd column), the difference between final and initial kinetic energy (3'rd column) and the degree of excitation of the p- basis coefficients ( 4 'th column) for different charges. The numbers (in brackets) mean that in $10 \%$ of the events that value was exceeded.

\begin{tabular}{|c||c|c|c|}
\hline$Z$ & $\left\langle E_{\text {coul }}\right\rangle(\mathrm{MeV})$ & $\left\langle\Delta E_{\text {kin }}\right\rangle(\mathrm{MeV})$ & $\left\langle 1-\left|c_{s}\right|\right\rangle$ \\
\hline 1 & $0.21(0.35)$ & $0.15(0.25)$ & $0.004(0.01)$ \\
3 & $1.9(3.2)$ & $1.3(2.5)$ & $0.03(0.09)$ \\
5 & $5.2(9.0)$ & $3.7(7.0)$ & $0.08(0.2)$ \\
\hline
\end{tabular}

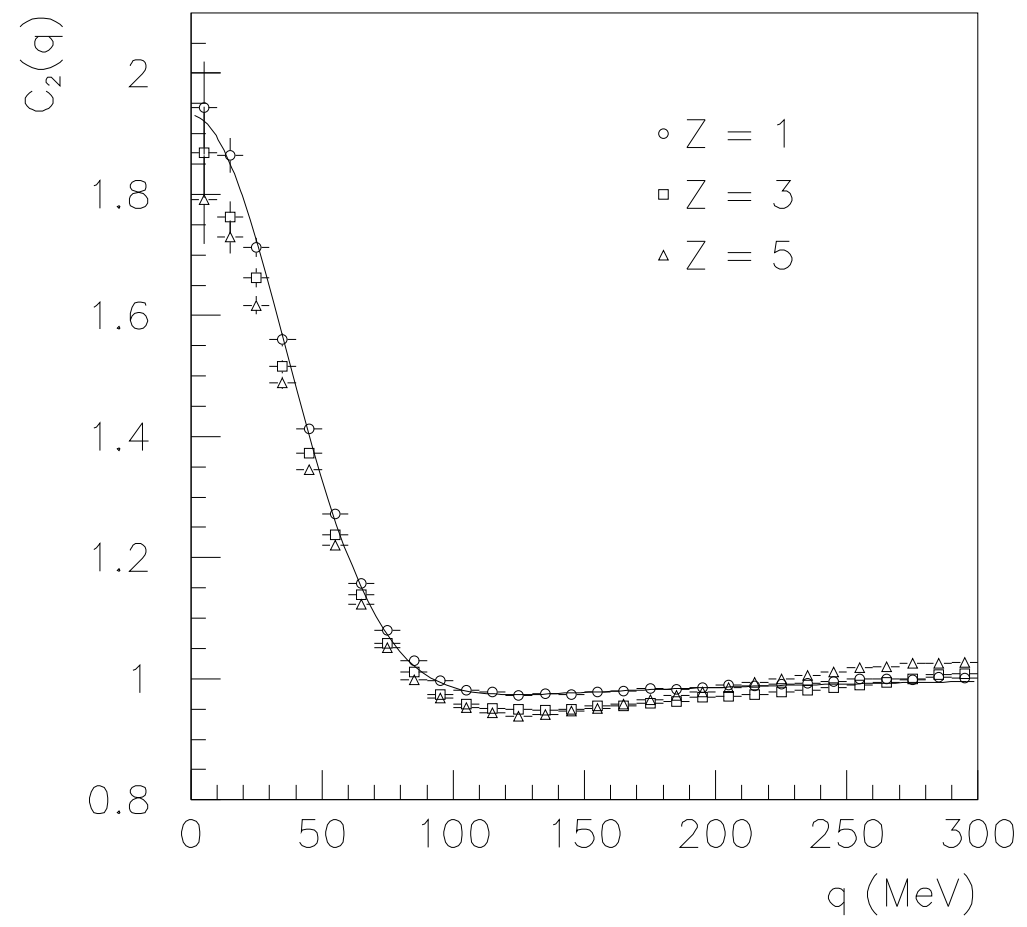

Figure 1: The 2- particle correlation functions for different charges. The solid curve is the theoretical result for noninteracting pions Eq. (11). 


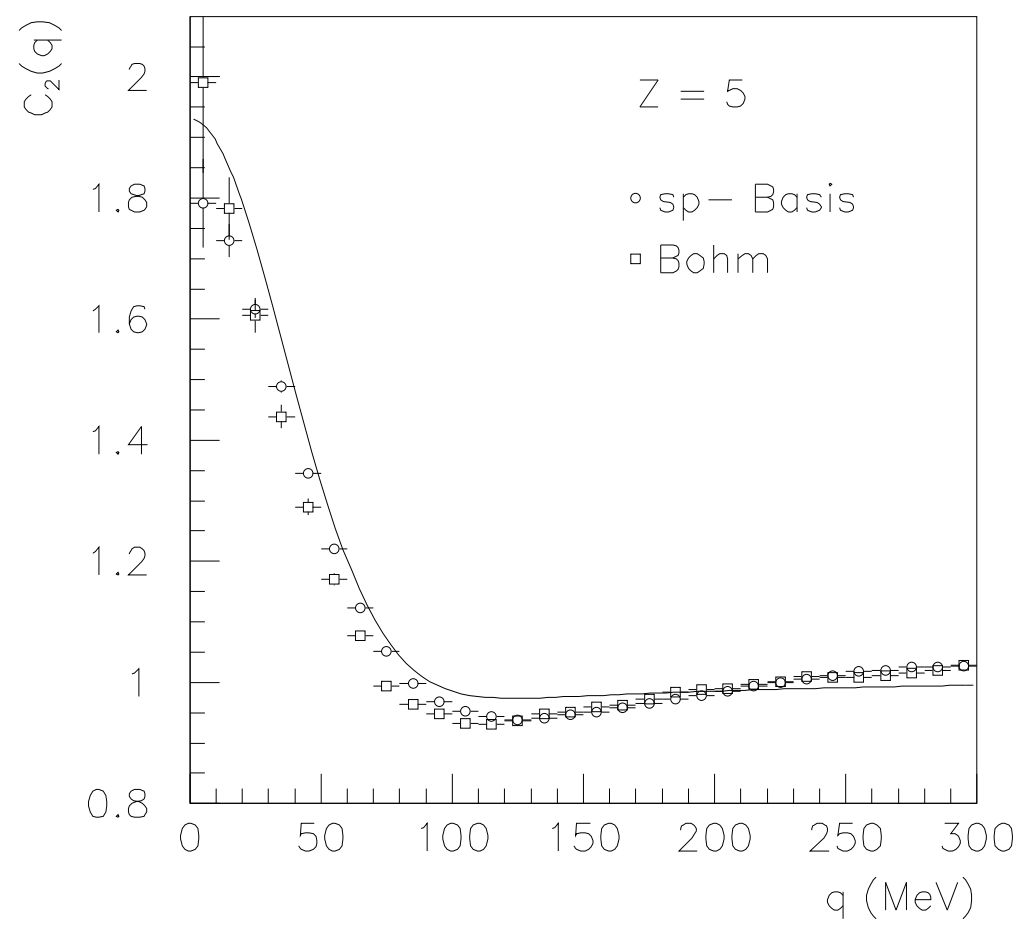

Figure 2: Correlation function for $Z=5$ and two different numerical methods. The solid curve is the theoretical result for noninteracting pions Eq. (11). 